\title{
Intermediate Mass Fragments production at low energy: reaction mechanism and isospin influence
}

\author{
Sara Pirrone ${ }^{1, *}$, Giuseppe Politi ${ }^{2,1}$, Brunilde Gnoffo $^{2,1}$, Enrico De Filippo ${ }^{1}$, Paolo Russotto ${ }^{3}$, Giuseppe Cardella ${ }^{1}$, \\ Elena Geraci ${ }^{2,1}$, Luigi Lo Monaco ${ }^{2}$, Nunzia Simona Martorana ${ }^{2,3}$, Angelo Pagano ${ }^{1}$, Emanuele Vincenzo Pagano ${ }^{3}$, Ernest \\ Piasecki $^{4}$, Francesca Rizzo $^{2,3}$, Antonio Russo ${ }^{3}$, and Marina Trimarchi ${ }^{5,1}$ \\ ${ }^{1}$ INFN, Sezione di Catania -Catania, Italy \\ ${ }^{2}$ Dipartimento di Fisica e Astronomia "Ettore Majorana", Università degli Studi di Catania - Catania, Italy \\ ${ }^{3}$ INFN, Laboratori Nazionali del Sud- Catania, Italy \\ ${ }^{4}$ Heavy Ion Laboratory, University of Warsaw, Warsaw, Poland \\ ${ }^{5}$ Dipartimento di Scienze Matematiche e Informatiche, Scienze Fisiche e Scienze della Terra, Università, Messina - Messina, Italy
}

\begin{abstract}
In this work, we present a recent study concerning the break-up of the Projectile-Like (PLF) into two fragments, following more violent deep-inelastic collisions in the reactions ${ }^{78} \mathrm{Kr}+{ }^{40} \mathrm{Ca}$ and ${ }^{86} \mathrm{Kr}+{ }^{48} \mathrm{Ca}$ at $10 \mathrm{~A} \mathrm{MeV}$, realized at INFN-Laboratori Nazionali del Sud by using the $4 \pi$ multi detector CHIMERA. For these systems, we have already analyzed the fusion-evaporation and fission-like processes. A selection method has been developed, in order to discriminate PLF break up events from those due to other mechanisms which populate the same region of the phase- space. A preference for PLF aligned break-up, along the direction of the PLF-TLF separation axis with the light fragment emitted in the backward part, has been put in light, suggesting the presence of dynamical effects. Also a comparison between the neutron-rich ${ }^{86} \mathrm{Kr}+{ }^{48} \mathrm{Ca}$ and neutron-poor ${ }^{78} \mathrm{Kr}+{ }^{40} \mathrm{Ca}$ systems is presented, to put in evidence the isospin role in these processes.
\end{abstract}

\section{Introduction}

The energy domain $\mathrm{E} / \mathrm{A} \leq 15 \mathrm{AMeV}$, the so called low enery regime, is characterized from the competition between the Compound Nucleus formation and its consequent deexcitation through fusion-fission and fusion-evaporation, and other binary processes like quasi elastic scattering and deep inelastic collisions.

These reaction mechanisms populate a wide range of mass region that include Light Charged Particles, Fission Fragments, Evaporation Residues and Intermediate Mass Fragments $(\mathrm{Z} \geq 3)$.

Mainly the reaction mechanisms responsible of the IMF production are the fission - like processes and the damped deep inelastic collision followed by projectilelike break -up. These processes, at this energy, populate the same region of the phase space, and in order to disentangle among them, a careful selection method has to be developed.

We have already studied the fusion-evaporation and fission-like processes in the reactions ${ }^{78} \mathrm{Kr}+{ }^{40} \mathrm{Ca}$ and ${ }^{86} \mathrm{Kr}+{ }^{48} \mathrm{Ca}$ at $10 \mathrm{AMeV}[1-4]$ with the $4 \pi$ multidetector CHIMERA [5-7].

In particular, considering the mass, charge and angular distributions of the reaction products, well identified by CHIMERA detector, we were able to measure the evaporation residues and fission-like cross sections for the two reactions. The comparison between the two systems, that

*e-mail: sara.pirrone@ct.infn.it differ by 16 neutrons, the maximum difference in neutrons number achievable by using these stable nuclei, put in light that the neutron enrichment seems to limit the formation of the composite system and to inhibit the fission decay channel.

A brief summary of the main results of this analysis are presented. Then we are going to present a selection method for the analysis of projectile-like break-up events. We would like to point out that in previous experiments performed at higher energy, it was observed an evident influence of the isospin on the nature of the break-up of the PLF [8-10], and now we explore if also at low energy the isospin plays a crucial role.

\section{Fusion-evaporation and fission-like processes}

The capability of detection and the high accuracy of the measurements obtained by using the CHIMERA array, allowed us to identify and kinematically distinguish the reaction products coming from different reaction mechanisms[1].

In particular, we were able to separate the evaporation residues and the fission like fragments coming from the decay of the compound nucleus, as it is shown in Fig.1, in which are reported the results obtained selecting "complete events" for the two systems. We define "complete events", those selected by imposing the condition of a total measured momentum, $P_{\text {tot }} \geq 0.6 P_{\text {beam }}$, where $P_{\text {beam }}$ 

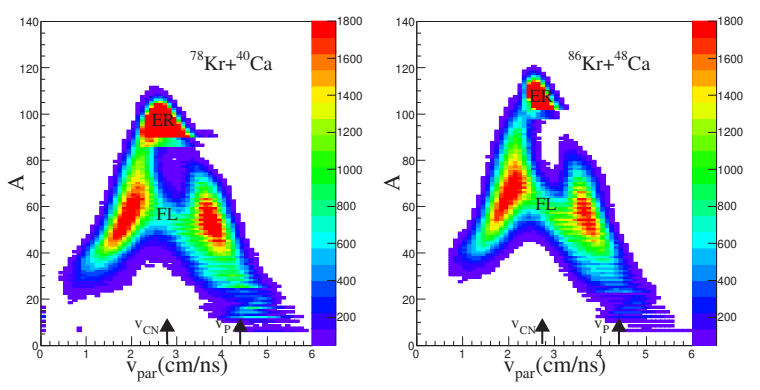

Figure 1. Mass versus parallel velocity for reaction products detected in "complete events" for the two systems; the arrows indicate compound nucleus $\left(v_{C N}\right)$ and projectile velocity $\left(v_{P}\right)$. (Color scale of the $\mathrm{Z}$-axis is in relative units).

is the total beam momentum, and a total detected mass $A_{\text {tot }} \geq 0.75 A_{C N}$, for each system.

In this figure the mass $A$ versus the parallel velocity $v_{\text {par }}$ is reported for each reaction product and for the two studied systems; we can distinguish the fragments coming from the different decay modes of the composite system formed in the reactions, as the Evaporation Residues (ER with $90 \leq A \leq 110$, for the neutron poor system and $100 \leq A \leq 120$ for the neutron rich one) centered around the compound nucleus velocity $v_{C N}$ and the fission-like fragments (FL with $10 \leq A \leq 85$ for the neutron poor system and $10 \leq A \leq 95$ for the neutron rich one) distributed along the two branches, coming from the corresponding possible kinematic solutions.

For the two systems, we built the angular distributions of the IMF, and by their integration, and by using the study of the Rutherford and elatic scattering, we calculated the production cross sections for each fragment. The charge distribution is presented in Fig.2.

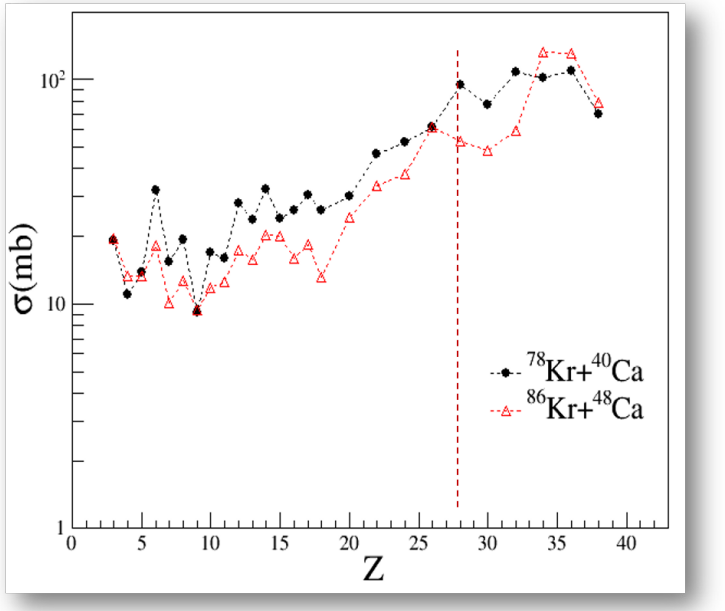

Figure 2. Charge distributions for the two systems

We note that for both systems the distribution presents a staggering effect, more evident for the neutron poor system, and that is not symmetric with respect to $Z=26 / 28$, that indicates a presence of a non equilibrated component of the production mechanism of the heavier fragments.

Table 1. Evaporation residues, fission-like, fusion and total quarter point reaction cross sections for the two reactions.

\begin{tabular}{ccccc}
\hline \hline & $\begin{array}{c}\sigma_{E R} \\
(\mathrm{mb})\end{array}$ & $\begin{array}{c}\sigma_{F L} \\
(\mathrm{mb})\end{array}$ & $\begin{array}{c}\sigma_{\text {fus }} \\
(\mathrm{mb})\end{array}$ & $\begin{array}{c}\sigma_{\text {reac }}^{q p} \\
(\mathrm{mb})\end{array}$ \\
\hline${ }^{78} \mathrm{Kr}+{ }^{40} \mathrm{Ca}$ & $455 \pm 70$ & $850 \pm 120$ & $1305 \pm 190$ & $2390 \pm 250$ \\
${ }^{86} \mathrm{Kr}+{ }^{48} \mathrm{Ca}$ & $400 \pm 60$ & $530 \pm 85$ & $930 \pm 145$ & $2520 \pm 260$ \\
\hline \hline
\end{tabular}

In the Table 1 is reported the measured cross sections for different reaction mechanisms and for both systems. From these results we can deduce that the fusion-fission process is favoured for the neutron poor system. Besides, looking at the difference $\sigma_{\text {reac }}^{q p}-\sigma_{\text {fus }}$, we note that this quantity is bigger for the neutron rich system, meaning a larger contribution of non equilibrated reactions in this case.

\section{Project-like break-up processes}

To discriminate PLF coming from the break-up events from the other processes which populate the same region in phase space, first of all we have to select events with three IMF in the exit channel. In Fig. 3 is shown, for this class of events, the correlation between the fragment mass and parallel velocity of the reaction products in the case of the ${ }^{78} \mathrm{Kr}+{ }^{40} \mathrm{Ca}$ system.

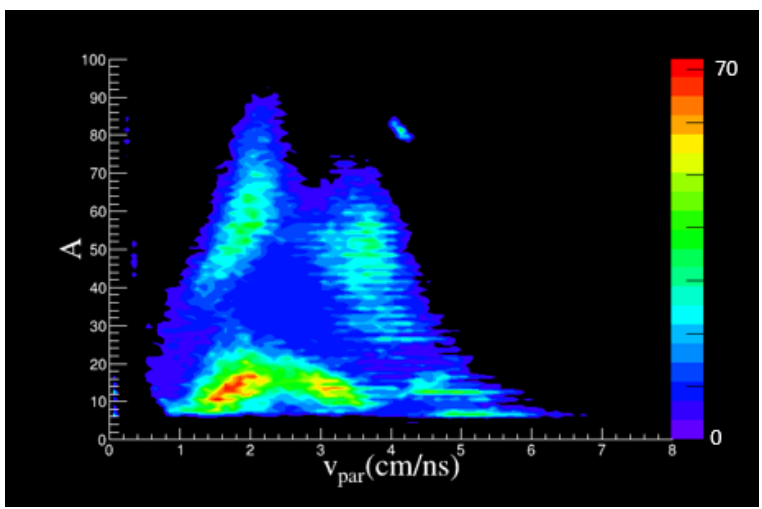

Figure 3. Correlation between the fragment mass and parallel velocity of the reaction products in events with 3 IMFs well detected and identified

Different mechanisms populate this plot: the break-up of the slow fission fragment from Compound Nucleus, the break-up of the fast fission fragment from Compound $\mathrm{Nu}$ cleus and the break-up of the PLF.

In fact, after its formation the Compound Nucleus can decay through fission and then the slow fission fragment can break-up into two fragments. At the end of this process we have three body: the fast fragment emitted in the fission of the Compound Nucleus $\left(F_{1}\right)$ and the two fragments produced in the break-up of the slow- fission fragment $\left(F_{2}^{I} a n d F_{2}^{I I}\right)$, for threshold reasons, in the kinematical solution where the lighter fragment is forward emitted. 
Similarly, if after the fission of the Compound Nucleus the fast fragment produced breaks up into two fragments, at the end we observe: the slow fragment emitted in the fission of the Compound Nucleus $\left(F_{2}\right)$ and the two fragments produced in the break-up of the fast- fission fragment $\left(F_{1}^{I}\right.$ and $F_{1}^{I I}$ ), with the lighter fragment backward or forward emitted.

Finally, the break-up of the projectile mechanism can occur. This mechanism is originated from a a two step mechanism, compatible with the hypothesis of a binary deep inelastic interaction, followed by the further fissionlike decay of the PLF. The three body produced in this reaction are: a part of the Target-Like Fragment $\left(T^{*}\right)$ and the two fragment coming from the break-up of the PLF ( $P^{I}$ and $P^{I I}$ ), with the lighter backward or forward emitted.

In Fig.4 is reported the reconstructed source of each combination of two fragments produced in events with 3 IMFs. They are the slow fragment emitted in the fission of the Compound nucleus $\left(F_{2}\right)$ or the $\operatorname{PLF}\left(P^{*}\right)$ or the fast fission fragment $\left(F_{1}\right)$.

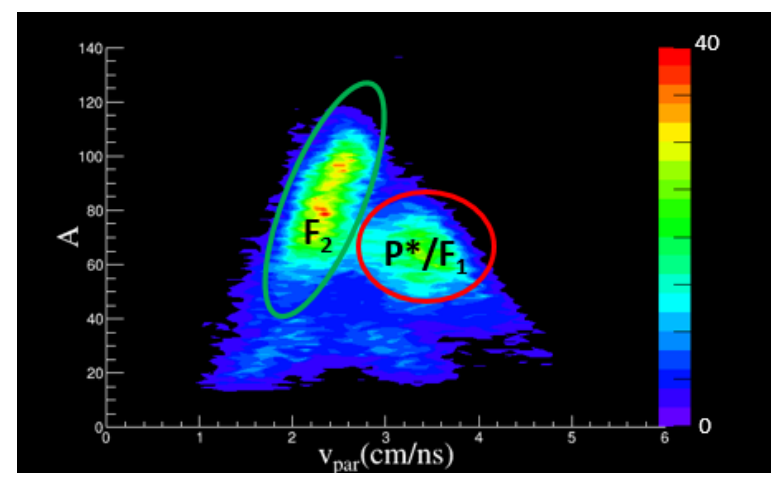

Figure 4. The mass versus parallel velocity plot of the reconstructed source of each combination of two fragments in events with 3 IMFs well detected and identified.

In order to be sure to take into account only events due the PLF break-up, we required that the reconstructed source of two fragments is $\left(P^{*}\right)^{*} /\left(F_{1}\right)$ and that the third fragment does not belong to the slow branch of fission from Compound Nucleus.

The figures are relative to the ${ }^{78} \mathrm{Kr}+{ }^{40} \mathrm{Ca}$ system, but similar results were obtained for the ${ }^{86} \mathrm{Kr}+{ }^{48} \mathrm{Ca}$ system. The correlation between the mass and the parallel velocity of the fragments produced int the PLF break-up, is shown in Fig. 5 on the left for ${ }^{78} \mathrm{Kr}+{ }^{40} \mathrm{Ca}$ and on the right for the ${ }^{86} \mathrm{Kr}+{ }^{48} \mathrm{Ca}$ system.

Very important information about the nature of the break-up process can be extracted from the angular distribution in the PLF reference frame. A main signature of a dynamical break- up is an alignment of the break- up axis with the separation direction of the two primary fragments (PLF and TLF), with the lighter breakup fragment backward emitted. The aligned break-up demonstrates the persistence of the memory of the previous deep inelastic step and in particular of the direction of the separation axis between PLF and TLF.

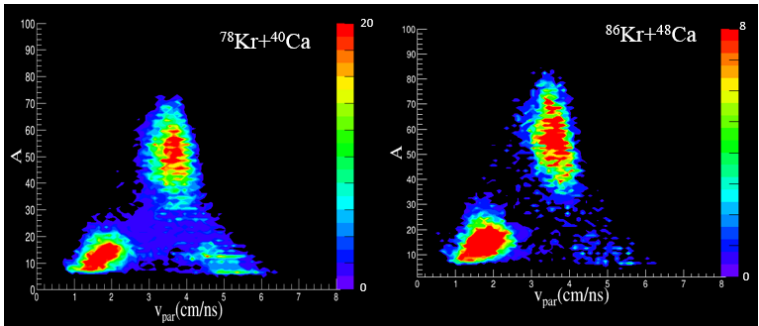

Figure 5. Mass versus parallel velocity lot for the PLF break-up on the left for ${ }^{78} \mathrm{Kr}+{ }^{40} \mathrm{Ca}$ and on the right for the ${ }^{86} \mathrm{Kr}+{ }^{48} \mathrm{Ca}$ system.

A very nice observable, that allows to disentangle between statistical and dynamical break-up, is the cosine of $\theta$ proximity. The proximity angle is defined as the angle between the separation axis between PLF and TLF and the break-up axis oriented from the Light to the Heavy fragment.

The distributions in $\theta$ proximity for different asymmetry parameters, $A_{H} / A_{L}$, and for the two systems, are reported in Fig. 6.

In these distributions, we observe the presence of two components, one isotropic, coming from relaxed process, and the other one, peaked around 1, with aligned fragments, suggesting dynamical effects. Dynamical break-up component prevails for the neutron rich system, in agreement with the results obtained in previous experiments performed at higher energy[8-10].

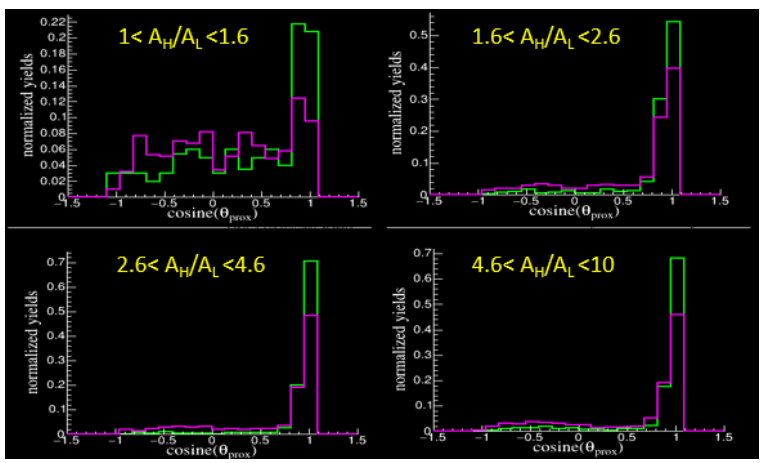

Figure 6. Distribution in cosine of $\theta$ proximity for the two studied systems for different asymmetry parameters.

\section{Conclusion}

Analisys relative to the IMF production in the reactions ${ }^{78} \mathrm{Kr}+{ }^{40} \mathrm{Ca}$ and ${ }^{86} \mathrm{Kr}+{ }^{48} \mathrm{Ca}$ were presented. In particular we reported the obtained results from the Compound nucleus reactions, with production of fission like fragments, evaporation residues and light charge particles. Then we presented a selection method, in order to discriminate PLF break-up from events due to other mechanisms, fusionfission like processes, which populate the same region of the phase-space. A preference for PLF aligned break-up, along the direction of the PLF-TLF separation axis with 
the light fragment emitted in the backward part, has been evidenced, suggesting dynamical-non equilibrium effects. The results seem to put in evidence that the dynamical effects are more pronounced for the reactions ${ }^{86} \mathrm{Kr}+{ }^{48} \mathrm{Ca}$, respect to ${ }^{78} \mathrm{Kr}+{ }^{40} \mathrm{Ca}$ one, for the different asymmetry parameters, in agreement with the results obtained at higher energy.

\section{References}

[1] S.Pirrone et al., Eur. Phys. J.55, 22 (2019)

[2] B.Gnoffo, Il Nuovo Cimento C,39 275 (2016)
[3] S.Pirrone et al., Journal of Physic: Conf. Series 515 012018 (2014)

[4] G.Politi et al., JPS Conf. Proc.6 030082 (2015)

[5] A.Pagano et al., Nucl. Phys. A 681331 (2001)

[6] G.Politi et al., IEEE NSS Conference Record N28-5 1140 (2005)

[7] M.Alderighi et al., IEEE Transactions on Nuclear Science 53279 (2006)

[8] E.De Filippo, A.Pagano, Eur. Phys. J. A50 32 (2014)

[9] P.Russotto et al., Phys. Rev. C 81064605 (2010)

[10] P.Russotto et al., Phys. Rev. C 91014610 (2015) 